\title{
Acompanhamento de Alunos em Ambientes Virtuais de Aprendizagem Baseado em Sistemas Tutores Inteligentes
}

\author{
Daniel Cirne Vilas-Boas dos Santos ${ }^{1}$, Taciana Pontual Falcão ${ }^{1}$ \\ ${ }^{1}$ Departamento de Estatística e Informática - Universidade Federal Rural de \\ Pernambuco (UFRPE) \\ Rua Dom Manoel de Medeiros, S/N - Dois irmãos - Recife - PE - Brazil \\ daanielvbegmail.com; taciana.pontualeufrpe.br
}

\begin{abstract}
Virtual learning environments (VLE) are getting mature as teaching and learning platform in distance education, being used by most of the educational institutions in Brazil. In these environments tutors have a fundamental role for supporting students in their activities and monitoring their progress. However, tutors face difficulties to perform their work with efficacy, given the high number of students in VLEs and the load of assignments. This paper presents a tool prototype aiming at support tutors' work in VLEs, using classification and grouping algorithms that can provide an enhanced view of students' progress and behavior within the environment

Resumo. Os ambientes virtuais de aprendizagem (AVAs) se consolidaram como plataforma de ensino e aprendizagem a distância mais utilizada no país. Nestes ambientes, os tutores assumem um papel de grande importância no acompanhamento dos alunos e apoio na realização de atividades. Entretanto, os tutores enfrentam dificuldades para realizar tal acompanhamento de forma eficaz, dados o grande número de alunos e o volume de atividades. Este artigo apresenta o protótipo de uma ferramenta que objetiva apoiar o trabalho dos tutores em AVAs, utilizando algoritmos de classificação e agrupamento que podem fornecer uma visão aprimorada do desempenho e comportamento dos alunos na plataforma.
\end{abstract}

\section{Introdução}

A Educação a Distância (EaD) surgiu como modalidade de ensino em conjunto com a criação de meios de comunicação capazes de conectar pessoas em localidades distintas, e foi evoluindo junto com eles. Desde seu surgimento (educação por correspondência) até o momento atual, houve diversas modificações e regulamentações que culminaram com o modelo vigente no Brasil, predominantemente baseado em sofisticados ambientes virtuais de aprendizagem (AVAs) acessíveis na Internet, e tendo como principais atores alunos, professores e tutores [Machado e Machado 2004].

A tutoria é considerada um dos papéis fundamentais para a boa execução do ensino a distância. $\mathrm{O}$ aumento do número de alunos em turmas de $\mathrm{EaD}$ e o aparecimento de plataformas de ensino online massivas (MOOC) tornaram inviável o acompanhamento de muitos alunos por poucos professores, o que deu maior autonomia para os tutores agirem diretamente no andamento do curso, tendo papel de orientador pedagógico, tecnológico e motivacional [Mill et al. 2010]. Atualmente, o tutor exerce 
VI Congresso Brasileiro de Informática na Educação (CBIE 2017)

Anais do XXVIII Simpósio Brasileiro de Informática na Educação (SBIE 2017)

papel fundamental em sistemas educacionais a distância, pois é responsável por acompanhar, supervisionar e manter o progresso dos alunos durante todo o processo de aprendizado. Para isso, os tutores devem dar o suporte e estímulo necessários para incentivar o aprendizado dos alunos, mantendo a maior proximidade possível e fazendo assim com que os questionamentos e dificuldades enfrentadas durante o curso possam ser mais facilmente resolvidos [Preti 2002, Machado e Machado 2004].

Porém, os tutores enfrentam várias dificuldades, principalmente no acompanhamento dos alunos. A criação de uma supervisão mais pessoal em um ambiente social online é um dos grandes desafios das plataformas de ensino atuais. A responsabilidade de diminuir a impessoalidade das relações recai sobre o tutor [Higley 2014], que precisa de capacitação profissional e de ferramentas nos AVAs que lhe permitam acompanhar melhor e dar um melhor feedback aos aprendizes. Sendo o AVA a principal ferramenta de comunicação entre tutores e alunos, é necessário que esses ambientes forneçam subsídios para mitigar os problemas causados pela distância temporal e física característica da modalidade de EaD. Apesar da constante evolução nos mecanismos tecnológicos que dão suporte à atividade de tutores e alunos nos AVAs, ainda existem diversos problemas que são enfrentados diariamente na interação - vários relacionados ao comportamento humano e à estruturação dos cursos e seus materiais, como: falta de capacitação profissional; má construção dos materiais didáticos; falta de disciplina dos estudantes (que precisam se adaptar a uma nova metodologia depois de anos no ensino tradicional, resultando num comportamento de pouco acesso); e dificuldade de acompanhamento e estudo em ambientes online [Morgado 2003].

Com um enfoque na perspectiva dos tutores, esse trabalho tem como propósito compreender melhor os problemas enfrentados por eles em AVAs, especialmente no que diz respeito ao acompanhamento dos alunos, para criar uma proposta de ferramenta que possa solucionar ou mitigar as dificuldades identificadas, visando melhorias no processo de ensino e aprendizagem na EaD. Com base em entrevistas com tutores e na literatura, foi proposta uma ferramenta que usa técnicas de Inteligência Artificial, que vêm apresentando resultados promissores em contextos educacionais. Em particular, a proposta é fundamentada em conceitos de Sistemas Tutores Inteligentes (STIs), que são plataformas de aprendizado online que simulam o comportamento de tutores humanos, por meio da observação das atividades dos alunos dentro do ambiente, associadas a uma base de conhecimento e algoritmos de decisão [Gavidia e Andrade 2013].

Este artigo está organizado da seguinte forma: a Seção 2 apresenta os Sistemas Tutores Inteligentes e a associação de STIs com a tutoria humana; na Seção 3 é descrita a metodologia utilizada neste trabalho; a Seção 4 apresenta os resultados das entrevistas realizadas com tutores; a Seção 5 descreve a solução proposta e a sua avaliação junto aos tutores; e por fim, a Seção 6 traz as conclusões e trabalhos futuros.

\section{Sistemas Tutores Inteligentes}

Os sistemas tutores inteligentes (STIs) surgiram na década de 80 com o aparecimento dos primeiros conceitos de Inteligência Artificial (IA) [Gavidia e Andrade 2013] e ganharam bastante espaço na comunidade científica e no mercado nos últimos anos, principalmente devido ao seu potencial de permitir que uma vez desenvolvido, o 
VI Congresso Brasileiro de Informática na Educação (CBIE 2017)

Anais do XXVIII Simpósio Brasileiro de Informática na Educação (SBIE 2017)

sistema possa funcionar relativamente autônomo. Esses sistemas têm uma maior flexibilidade de adaptação às necessidades da sociedade atual, cada vez mais dinâmica e heterogênea, que precisa da informação de maneira rápida e adaptável. Desde seu surgimento, os STIs evoluíram em diversos aspectos técnicos e pedagógicos e atualmente são usados em contextos educacionais e profissionais [Gavidia e Andrade 2013].

A principal abordagem utilizada pelos STIs na área educacional é observar o comportamento e as ações realizadas pelos alunos dentro de um sistema, engajando-os a resolverem atividades por meio do aprender fazendo. A partir da observação e coleta desses dados, técnicas de IA, como por exemplo redes bayesianas para dedução de premissas sobre os alunos [Friedman et al. 2000] e árvores de decisão para guiá-los por uma trilha de aprendizagem [Cha et al. 2006], são aplicadas, oferecendo conteúdos e sugestões para que as dificuldades do aluno sejam sanadas.

Apesar das definições tradicionais de STIs sugerirem que eles devem ter o comportamento semelhante ao de um tutor humano, os problemas educacionais atuais ainda carecem de soluções tecnológicas que ajudem a superá-los [Giraffa e Viccari 1997] [Santos e Jorge 2013]. Mesmo com o grande avanço tecnológico, os STIs não são capazes de substituir professores e tutores em atividades pedagógicas. Resultados promissores têm sido encontrados ao utilizar sistemas inteligentes associados à mediação de professores e tutores. Rumble (2003) e Giraffa e Viccari (1997) argumentam que os STIs poderiam ser mais efetivos caso incorporassem aspectos de ensino supervisionado por professores e tutores, permitindo a intervenção tanto de humanos quanto da máquina em busca de melhores resultados. Pesquisas recentes sobre STIs sugerem que eles adotem princípios da $\mathrm{EaD}$, mais especificamente o ensino pela internet (e-Learning) e aprendizado supervisionado por tutores humanos [Oliveira 2012]. Também seguindo essa linha de raciocínio, Bittencourt e Costa (2009) defendem a integração entre STIs e ambientes e-Learning, dado que a fusão combinaria características importantes de ambos, como ensino de múltiplos domínios, suporte colaborativo e intervenção pedagógica humana permeados por técnicas de IA.

Desta forma, percebe-se um grande potencial na combinação de sistemas inteligentes e mediação humana, pois além de aumentar a motivação dos alunos e facilitar o trabalho de tutores, também é possível propor alternativas para problemas típicos da EaD. Muitas dessas dificuldades vêm sendo tratadas pela mineração de dados (MD) voltada para educação, que se concentra na aplicação de recursos computacionais para detectar padrões em grandes volumes de dados relacionados ao aprendizado, que não seriam possíveis de analisar manualmente devido ao grande volume [Romero e Ventura 2013]. Algumas das principais abordagens para solução de problemas por meio da MD são: predição, agrupamento e descoberta de modelos a partir dos conjuntos de dados [Baker 2010]. No presente trabalho, são utilizados os algoritmos de agrupamento e classificação Random Forest e Expectation Maximization, para auxiliar tutores a superarem as dificuldades de acompanhamento de alunos em AVAs.

\section{Metodologia}

\subsection{Entrevistas com Tutores}


VI Congresso Brasileiro de Informática na Educação (CBIE 2017)

Anais do XXVIII Simpósio Brasileiro de Informática na Educação (SBIE 2017)

Para conhecer de forma mais detalhada os problemas enfrentados por tutores, foram realizadas entrevistas semi-estruturadas, durando de 25 a 45 minutos e gravadas em áudio, com 9 tutores (Tabela 1).

Tabela 1. Perfis dos tutores entrevistados

\begin{tabular}{|l|l|l|l|}
\hline Entrevistado & Escolaridade & Área de formação & Experiência com tutoria online \\
\hline E1 & Doutorado & Educação Ambiental & 4 a 5 anos \\
\hline E2 & Doutorado & Ciência da Computação & 6 anos \\
\hline E3 & Doutorado & Ciência da Computação & 5 anos \\
\hline E4 & Pós-graduação & Tecnologia da Informação & 7 anos \\
\hline E5 & Doutorado & Ciência da Computação & 4 anos \\
\hline E6 & Graduação & Publicidade & 9 anos \\
\hline E7 & Mestrado & Letras & 7 anos \\
\hline E8 & Graduação & Publicidade & 3 anos \\
\hline E9 & Pós-graduação & Pedagogia & 3 anos \\
\hline
\end{tabular}

O roteiro incluiu: identificação da experiência e área de atuação de cada profissional; plataformas de ensino em que trabalharam e nível de satisfação com os recursos e capacitação oferecidas; rotina de trabalho e principais atividades; como eles realizam o acompanhamento de seus alunos; percepções sobre sua atuação e a evasão de alunos; principais problemas e desafios enfrentados; e informações relevantes sobre os alunos que podem ajudar no acompanhamento. A partir da análise dos dados coletados, foram levantados os principais problemas e necessidades que a ferramenta proposta deveria abordar, buscando resolver ou mitigar as deficiências e prover novos recursos.

\subsection{Desenvolvimento do Protótipo}

Para desenvolvimento do protótipo ${ }^{l}$ foram utilizadas as tecnologias Web HTML 5, CSS 3 e Javascript auxiliadas pelos frameworks AngularJS e Bootstrap executados sobre o automatizador de tarefas Gulp. Para representação das atividades dos alunos na plataforma, foi construída manualmente uma base de dados fictícia em formato csv incluindo as classificações previstas. A construção dessa base seguiu as etapas de análise do contexto e construção do modelo classificatório. Posteriormente a base foi separada em 3 conjuntos com 100 registros cada, de acordo com os padrões de classificação definidos, e foi convertida para o formato arff (Attribute-Relation File Format), padrão aceito pela ferramenta de análise de dados e aplicação de algoritmos WEKA (Waikato Environment for Knowledge Analysis) [Hall et al. 2009], que foi utilizado nas etapas de experimentação, escolha do algoritmo e concepção do protótipo.

\footnotetext{
${ }^{1} \mathrm{O}$ código fonte produzido nesta etapa se encontra disponível em https://github.com/daanielvb/tcc
} 
VI Congresso Brasileiro de Informática na Educação (CBIE 2017)

Anais do XXVIII Simpósio Brasileiro de Informática na Educação (SBIE 2017)

A partir dos atributos levantados por Gottardo et al. (2012) (possíveis características de alunos a serem obtidas em AVAs), da estrutura dos cursos de EaD, da possibilidade de extração de determinadas informações e das entrevistas com tutores, foram definidos 5 atributos (Tabela 2) a serem usados no protótipo.

Tabela 2. Atributos utilizados no modelo proposto

\begin{tabular}{|c|c|c|}
\hline Atributo & Unidade de medida & Descrição \\
\hline $\begin{array}{l}\text { Quantidade de acessos } \\
\text { por semana }\end{array}$ & $\begin{array}{l}\text { Número de acessos } \\
\text { semanais }\end{array}$ & $\begin{array}{c}\text { Número de acessos com duração acima de } 5 \\
\text { minutos durante a semana }\end{array}$ \\
\hline $\begin{array}{l}\text { Quantidade de } \\
\text { interações individuais }\end{array}$ & $\begin{array}{l}\text { Número absoluto de } \\
\text { interações }\end{array}$ & $\begin{array}{c}\text { Atividades realizadas individualmente, } \\
\text { como baixar material, responder questões, } \\
\text { enviar exercícios }\end{array}$ \\
\hline $\begin{array}{l}\text { Quantidade de } \\
\text { interações em grupo }\end{array}$ & $\begin{array}{l}\text { Número absoluto de } \\
\text { interações }\end{array}$ & $\begin{array}{l}\text { Atividades realizadas em conjunto, como } \\
\text { mensagem em fórum, atividades em grupo }\end{array}$ \\
\hline $\begin{array}{l}\text { Quantidade de aulas } \\
\text { assistidas }\end{array}$ & Número de aulas & $\begin{array}{l}\text { Vídeo-aulas assistidas ou material transcrito } \\
\text { /slides lidos }\end{array}$ \\
\hline $\begin{array}{l}\text { Média ponderada das } \\
\text { atividades }\end{array}$ & Média ponderada de 0 a 10 & $\begin{array}{c}\text { Média ponderada das atividades e exercícios } \\
\text { realizados online }\end{array}$ \\
\hline
\end{tabular}

A partir dos atributos e entrevistas, foram criados 3 padrões de classificação: i) necessidade de atenção, ii) possibilidade de evasão e iii) comportamento atípico. Segundo os profissionais entrevistados, alunos que ainda não visitaram o AVA ou que deixam de acessar após algum progresso dentro do curso precisam de atenção e por meio da ação do tutor é possível motivar esses alunos a continuar o curso. Já aqueles que tiraram notas baixas ou não entregaram atividades dentro dos prazos e deixaram de acessar o ambiente possuem uma probabilidade maior de evadirem da plataforma em definitivo. Alunos que não apresentam histórico de acesso aos materiais e aulas do curso e obtiveram boas notas nas atividades e avaliações ou que apresentam bom histórico de acesso e notas baixas das avaliações podem ser caracterizados com um comportamento atípico, pois a tendência é que quanto maior a presença do aluno no AVA maior seja sua compreensão sobre os assuntos abordados.

A partir das observações feitas e do contexto do problema, e resultados significativos ao aplicar os algoritmos presentes na ferramenta na base de dados construída, decidiu-se utilizar a árvore de decisão floresta aleatória (Random Forest) para classificar os alunos, criar um modelo e prever possíveis comportamentos futuros, por ser apontado como um dos melhores classificadores [Delgado et al. 2014]. Durante a avaliação da capacidade de generalização do modelo a validação cruzada com 10 folhas apresentou os melhores resultados. Também foi usado o algoritmo de clustering maximização de expectativas (EM), por conseguir realizar a tarefa de agrupamento sem a necessidade de conhecimento prévio da distribuição desses dados. O propósito deste algoritmo é separar os alunos em grupos de acordo com suas dificuldades e atividades realizadas na plataforma, permitindo ao tutor ter uma visão macro de sua turma de 
VI Congresso Brasileiro de Informática na Educação (CBIE 2017)

Anais do XXVIII Simpósio Brasileiro de Informática na Educação (SBIE 2017)

acordo com as características de sua escolha.

\subsection{Avaliação do Protótipo}

Para avaliar o protótipo construído foi elaborado um novo roteiro de entrevistas para ser aplicado com tutores. As entrevistas buscaram avaliar se a solução proposta atendia aos problemas identificados, se a interface apresentada era intuitiva e como aquela funcionalidades poderiam mudar o trabalho de tutoria, além de abrir espaço para críticas e sugestões. As entrevistas foram realizadas com 4 tutores (dos quais dois participaram da primeira fase de entrevistas: E1 e E5). Os outros dois tutores (EA e EB) tinham mestrado em Ciência da Computação e doutorado em Engenharia Mecânica e 1,5 e 6 anos de experiência na $\mathrm{EaD}$, respectivamente. As entrevistas tiveram duração aproximada de 30 minutos, e foram gravadas em áudio.

\section{Principais Resultados das Entrevistas com Tutores}

Os tutores têm a percepção que seu trabalho exerce uma grande influência sobre o estudo dos alunos, especialmente aqueles com maiores dificuldades: "Quando o tutor pontua individualmente, manda mensagem, isso influencia demais no aprendizado e ajuda bastante na não evasão" (E8); "A atividade do tutor influencia totalmente no aprendizado do aluno, tutores que não acompanham bem os alunos geram uma defasagem muito grande do nível geral da turma com relação ao conteúdo" (E2). Isso fortalece o propósito deste trabalho, que é melhorar o trabalho dos tutores, visando a construção de ambientes de ensino online mais eficientes.

O tempo de trabalho dos tutores nos AVAs foi de aproximadamente 14 horas semanais. Algumas das atividades realizadas nesse intervalo de tempo foram consideradas repetitivas ou pouco eficientes: "Eu tirava um dia da semana para verificar o relatório dos alunos, entrava de um a um e via os acessos e atividades que cada um realizou, daí caso alguém estivesse ausente durante a semana eu mandava uma mensagem perguntando o que havia acontecido" (E1).

Sobre os maiores problemas enfrentados, houve uma diversidade de opiniões. Foram citados problemas relacionados aos cursos e seus conteúdos, a forma engessada de execução da $\mathrm{EaD}$ em determinadas instituições de ensino, dificuldades no relacionamento e acompanhamento de alunos, e plágio. Em particular, a comunicação é considerada fundamental: "Para o aprendizado é necessário interagir, o diálogo é fundamental para aprender de fato." (E1); "Como tutor, sei que os alunos se sentem mais à vontade para conversar comigo do que com o professor, a interatividade é o coração do aprendizado nos ambientes de ensino online" (E7).

Nas entrevistas, os tutores foram expostos, por meio de uma explicação informal, ao potencial do uso de IA e mineração de dados como em STIs e seus possíveis resultados práticos na atividade diária do tutor. As reações à ideia foram positivas: "Acho que esse tipo de coisa deveria ser feito pra ontem e estar dentro de todos os ambientes de $\mathrm{EaD}$, a gente faz muita coisa que poderia ser feito pela máquina, (...) poderíamos focar ainda mais na parte do ensino enquanto a máquina faria um trabalho mais pesado" (E7); "Isso poderia ajudar muito meu trabalho, só de não precisar olhar aluno a aluno pra saber quem está precisando de atenção já me pouparia horas de trabalho, isso me deixaria menos cansado pra fazer outras coisas que às vezes não fica 
VI Congresso Brasileiro de Informática na Educação (CBIE 2017)

Anais do XXVIII Simpósio Brasileiro de Informática na Educação (SBIE 2017)

tão bom pelo cansaço" (E9).

\section{Protótipo Proposto}

O protótipo desenvolvido apresenta a visão de um AVA na perspectiva do tutor, com a inclusão de funcionalidades que dão maior flexibilidade para acompanhar os alunos e realizar intervenções. Para mitigar problemas relacionados à falta de conhecimento sobre os alunos e dificuldade de acompanhamento, foram utilizados algoritmos de mineração de dados e clusterização a partir de dados que seriam gerados pelos próprios alunos durante o uso do ambiente. A partir da aplicação das técnicas citadas são criados gráficos e relatórios para que o tutor possa compreender melhor as características da turma e de cada aluno detalhadamente. Ao clicar em determinada região dos gráficos, o tutor pode interagir diretamente com um grupo de alunos (Figura 1).

\section{Uso do sistema}

- Acessos Semanais - Interaçóes individuais $\bullet$ Interaçōes em grupo - Aulas Assistidas @ Média de acerto em questões

\begin{tabular}{ll|l} 
Acessos semanais $\quad$ B Buscar aluno Nome ou Email Acessos semanais
\end{tabular}

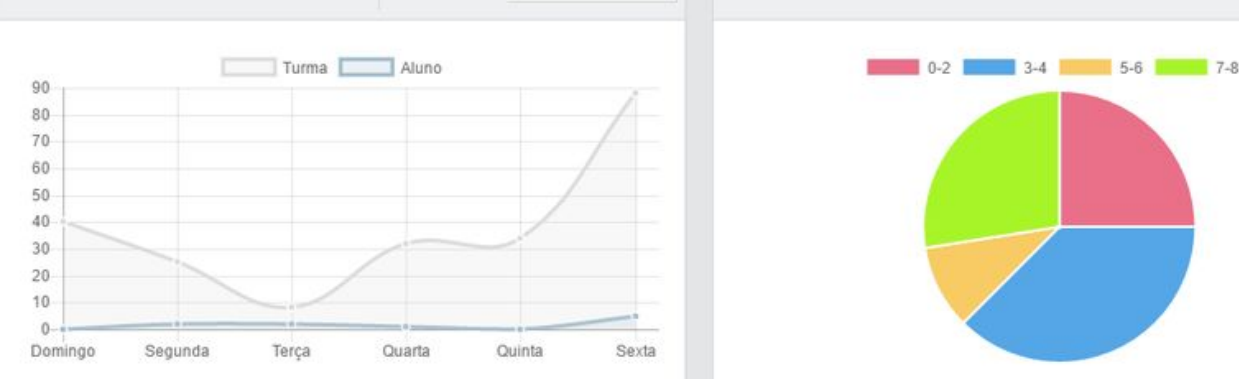

Figura 1. Interface de acompanhamento de uso

Para diminuir a execução de tarefas repetitivas pelos tutores, os alunos que demonstram dificuldades ou comportamentos em comum, como histórico de baixo acesso ou notas baixas em determinada atividade, podem ser agrupados de acordo com os atributos apresentados na Seção 3.2 (por meio do algoritmo EM, que roda a partir da ação do tutor, mas de forma transparente) e tratados pelos tutores de forma mais eficiente, diminuindo o tempo que seria gasto com o tratamento individual (Figura 2). Além disso, foi criado um modelo de classificação, que no protótipo se apresenta como uma funcionalidade de previsão de comportamento dos alunos, para que o tutor saiba, a partir de determinadas características de uso da plataforma e notas obtidas, que alguns alunos podem estar com dificuldades ou apresentando um comportamento de possível evasão, por exemplo. A partir da listagem das turmas, os alunos que estiverem dentro de grupos de atenção classificáveis pela ferramenta serão identificados (Figura 3).

Com base nas classificações dos alunos realizada pelos tutores dentro dos grupos que representam comportamentos que precisam de atenção, o sistema é capaz de criar um modelo de classificação baseado no algoritmo Random Forest. A partir dele, outros alunos ou novos padrões de comportamento para alunos antigos, que naturalmente mudam ao longo do tempo, podem ser indicados para determinada classificação, porém caberá ao tutor acatar ou não aquela sugestão, que posteriormente irá aperfeiçoar o próprio modelo resultando em modelos com maiores chances de acerto. 
VI Congresso Brasileiro de Informática na Educação (CBIE 2017)

Anais do XXVIII Simpósio Brasileiro de Informática na Educação (SBIE 2017)

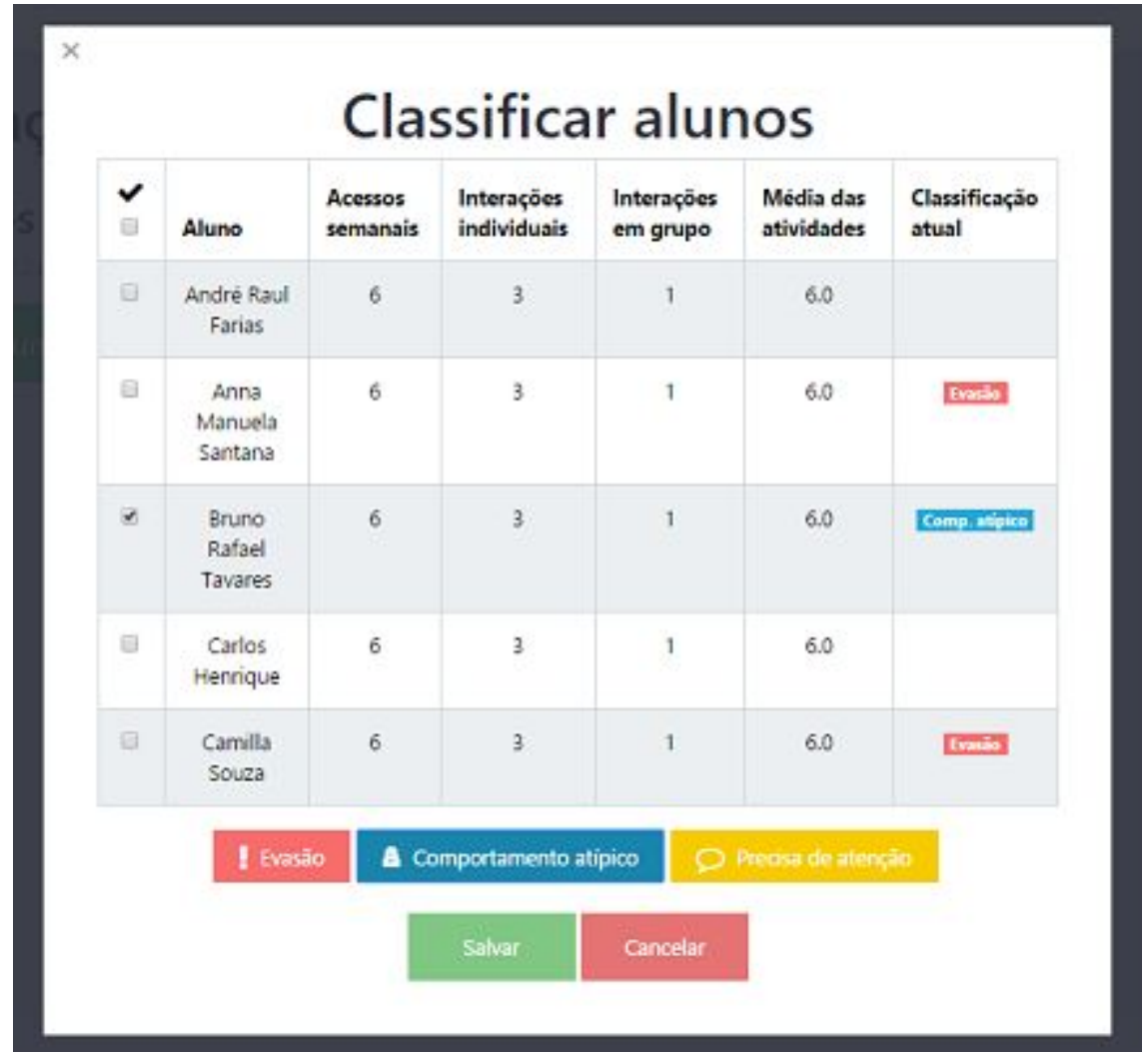

Figura 2. Interface de classificação após agrupamento dos alunos.

\section{Acompanhamento avançado}

\section{E Alunos da disciplina}

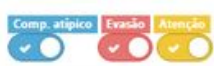

Aluno

Andre Raul Farias

Anna Manuela Santana

Bruno Rafael Tavare

Carlos Henrique

Camilla Souza

Dário Cunha

\begin{tabular}{|c|c|c|}
\hline Último acesso & Média & Indicadores \\
\hline 05/12/2016 19:30 & 6.0 & 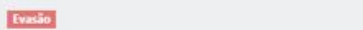 \\
\hline $09 / 12 / 201607: 30$ & 4.2 & Embia \\
\hline $11 / 12 / 2016$ 12:22 & 7.9 & Comportimento atpico \\
\hline $05 / 12 / 201622: 41$ & 0 & Atmex \\
\hline 05/12/2016 08:00 & 5.6 & \\
\hline 03/01/2017 08:11 & 9.6 & Gomportumento atpico \\
\hline
\end{tabular}

Figura 3. Interface de acompanhamento dos alunos por classificação.

\subsection{Avaliação do Protótipo}

Quatro principais funcionalidades do protótipo foram avaliadas: acompanhamento de notas, uso do sistema, agrupamento e classificação de alunos e modelo de classificação. Os tutores classificaram a exibição das notas por meio de gráficos como excelente ou boa: "Isso é realmente muito bom, nos ambientes que trabalhei as notas eram 
VI Congresso Brasileiro de Informática na Educação (CBIE 2017)

Anais do XXVIII Simpósio Brasileiro de Informática na Educação (SBIE 2017)

apresentadas em tabelas e não dava pra ter ideia de como estava a turma no geral (...) dá até para o tutor se auto avaliar, tipo quando muitos alunos tiram 8 porque erraram uma questão que valia 2, daí você pode pensar se a questão estava clara ou se o conteúdo não foi bem discutido." (EA); "Ao você visualizar aqueles alunos com notas baixas e pode clicar no gráfico e já ver quem são esses alunos, ainda mais podendo interagir com eles por meio das opções que você mostrou ali, seria maravilhoso." (EB).

A exibição dos dados de uso do ambiente pelos alunos teve sua interface classificada com excelente: "Isso é muito bom, pois geralmente a gente não tem quase nenhum dado dos nossos alunos, dessa forma a gente pode saber como os alunos se comportam na ferramenta, se estão acompanhando a disciplina e até repensar se estamos realmente trazendo os alunos pra perto por meio do nosso trabalho ou não." (E2). Com relação ao agrupamento e classificação dos alunos, os tutores se mostraram entusiasmados: "Após o tutor entender como funciona essa funcionalidade eu acho que tem um potencial muito grande. Dá pra você juntar os alunos de acordo com as características deles e intervir sobre aquele grupo específico." (E2); "É bom que você pode juntar os alunos que estão com poucos acessos e gravar eles com essa chance de evasão, podendo dar maior atenção a esses alunos para evitar que eles abandonem o curso." (EA). Já o E1 se mostrou interessado pela classificação de comportamento atípico: "Dá pra você acompanhar melhor aqueles alunos que estão se esforçando e com dificuldade nas atividades e também ficar de olho naqueles que podem estar copiando.". É importante ressaltar que todos os entrevistados afirmaram que nunca tinham visto uma funcionalidade desse tipo em um AVA e que seria um grande facilitador no acompanhamento dos alunos.

Por fim, os entrevistados concordaram que é necessário o modelo de classificação, pois realmente haveria muitos casos de mudança de comportamento e classificações desatualizadas: "Foi bem pensado, um aluno pode mudar de comportamento durante a semana e deixar de ser considerado como passível de evasão, dessa forma dá pra saber quantos alunos mudaram de comportamento ou até se foi classificado errado pela gente." (E2); "O interessante é que essa funcionalidade pode até prever pra gente quando um aluno apresenta um padrão de evasão ou atenção antes mesmo do tutor fazer o agrupamento." (EA).

\section{Conclusão e Trabalhos Futuros}

Esse trabalho teve como objetivo compreender melhor os desafios envolvidos na tutoria em AVAs para propor uma solução tecnológica que possa facilitar e contribuir para a eficácia do ensino e aprendizagem a distância. A partir de problemas identificados na literatura e em entrevistas com tutores, foi criado um protótipo com aplicação de técnicas de IA em AVAs com foco no acompanhamento e aproximação de tutores e alunos. O protótipo foi bem avaliado por tutores, constatando-se que existe um grande potencial de crescimento e inovação na associação de algoritmos de IA em ambientes educacionais e colaborativos, havendo diversas necessidades educacionais que podem ser atendidas por meio dessa área. Trabalhos futuros incluem a avaliação da solução proposta com um número maior de tutores; aprofundamento em um nicho mais específico de tutores e alunos; utilização de uma base de dados com um volume de dados significativo que represente mais fielmente o comportamento de alunos de $\mathrm{EaD}$; 
VI Congresso Brasileiro de Informática na Educação (CBIE 2017)

Anais do XXVIII Simpósio Brasileiro de Informática na Educação (SBIE 2017)

maiores estudos na escolha e validação dos algoritmos de classificação e agrupamento de dados a serem utilizados em AVAs; e o desenvolvimento de um AVA funcional ou plugin a partir do protótipo sugerido, que atualmente consiste apenas da interface.

\section{Referências}

Bittencourt, I. I. e Costa, E. (2009) "Modelos e ferramentas para a construção de sistemas educacionais adaptativos e semânticos". Tese (Doutorado) - DEE-UFCG, Campina Grande.

Baker, R. S. J. D. (2010) "Data mining for education.” International encyclopedia of education 7.3. p 112-118.

Cha, H. J. et al. (2006) "Learning Styles Diagnosis Based on User Interface Behaviors for the Customization of Learning Interfaces in an Intelligent Tutoring System", International Conference on Intelligent Tutoring Systems, Springer Berlin Heidelberg, v. 4053, p. 513- 524.

Delgado, M. F. et al. (2014) "Do we need hundreds of classifiers to solve real world classification problems?", J. Machine Learning Research, v. 15, n. 1, p. 3133 - 3181.

Friedman, N. et al. (2000) "Using Bayesian Networks to Analyze Expression Data", Journal of Computational Biology, v. 7, n. 3-4, p. $601-620$.

Hall, M. et al. (2009) “The WEKA Data Mining Software: An Update”, 1. ed. [S.1.].

Higley, M. (2014) "e-Learning: Challenges and Solutions, in E-learning industry", Disponível em: <http://elearningindustry.com/e-learning-challenges-and-solutions>. Acesso em: 13/09/2016.

Machado, L. D.; Machado, E. de C. (2004) “O papel da tutoria em ambientes de EAD”, In: Anais do XI Congresso Internacional de Educação a Distância.

Mill, D.; Oliveira, M. R. G. de; Ribeiro, L. R. de C. (2010) "Múltiplos enfoques sobre a polidocência na Educação a Distância virtual”, EdUFSCar, São Paulo, p. 13 - 22.

Morgado, L. (2003) Os novos desafios do tutor a distância: os novos desafios do tutor a distância: o regresso ao paradigma da sala de aula. Portugal.

Oliveira, P. P. de. (2012) "Um modelo de integração dos princípios de Sistemas Tutores Inteligentes e e-Learning a jogos do tipo MMORPG”, Dissertação (Mestrado) Programa de Pós-Graduação em Gestão do Conhecimento e da Tecnologia da Informação, Brasília.

Preti, O. (2002) “Apoio à aprendizagem: o orientador acadêmico", Cuiabá: [s.n.]. Disponível em: $<$ https://www.nescon.medicina.ufmg.br/biblioteca/imagem/2495.pdf>. Acesso em: 08/08/2016.

Romero, C. e Ventura, S. (2013) "Data mining in education". Wiley Interdisciplinary Reviews: Data mining and knowledge Discovery 3.1. p. 12-27.

Santos, G. e Jorge, J. (2013) "Atomic and Molecular Intelligent Tutoring Systems - A New Architecture for Interoperable Tutors as Open Educational Resources”, Proc. IEEE Int'1 Conf. Advanced Learning Technologies. 